\title{
New risk loci for JIA identified
}

A study by the international Juvenile Arthritis Consortium for Immunochip (JACI) has revealed many new risk loci associated with juvenile idiopathic arthritis (JIA). The findings increase understanding of the genetic basis of susceptibility to this disease and the mechanisms underlying its pathogenesis.

The study utilized the Immunochip genotyping array, which was custom designed to provide dense coverage of single-nucleotide polymorphisms in the HLA region and in 186 non-HLA regions confirmed as risk loci for at least one of 12 autoimmune diseases (not including JIA). 2,816 patients with JIA and 13,056 control individuals from across the USA, UK and Germany were included. To reduce heterogeneity, the analysis was restricted to patients with oligoarticular or rheumatoidfactor-negative polyarticular JIA, which account for around $70 \%$ of cases of JIA.

"We have confirmed and strengthened the associations with three established JIA risk loci, the HLA region, PTPN22 and PTPN2, and identified 14 JIA risk loci that reach genome-wide significance $\left(P<5 \times 10^{-8}\right)$ for the first time," states Anne Hinks, speaking on behalf of multiple members of JACI. "We also identified 11 loci with lower levels of association ( $P$ values between $1 \times 10^{-6}$ and $\left.5 \times 10^{-8}\right)$, which could potentially be true JIA susceptibility loci." On the basis of their data, the authors estimate that the HLA region and the 27 non-HLA loci with the highest association with the disease explain $13 \%$ and $6 \%$ of the risk of JIA, respectively.

Eight of the new loci associated with JIA were narrowed down to single genes. "One pathway is emerging as being crucial in JIA pathogenesis: the IL-2 pathway, which has key roles in T-cell activation and development, and maintenance of immune tolerance through regulation of regulatory T-cell function," says Hinks; associations with IL2-IL21, IL2RA

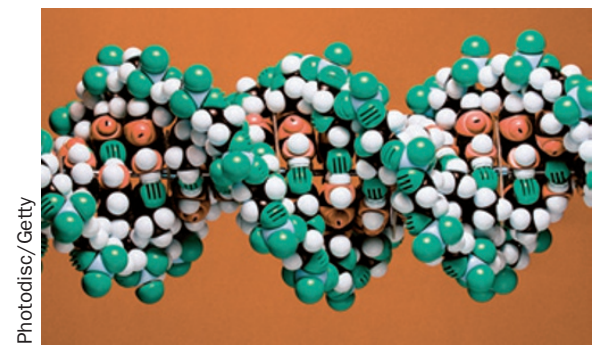

and $I L 2 R B$ all reached genome-wide significance, as did those with $\mathrm{SH} 2 \mathrm{~B} 3$ and STAT4, involved in T-cell activation and differentiation, respectively.

"An important outcome of the use of the Immunochip is the ability to place JIA genetics in the context of other autoimmune disease genetics," explains Hinks. The strength of association with JIA of only one region, C5orf56-IRF1, was different between the two disease categories examined, being stonger for oligoarticular JIA. Many of the identified risk loci for JIA were also shared by other autoimmune diseases, particularly rheumatoid arthritis, although the variant involved was not always the same for each disease. "Such comparisons and contrasts can motivate research on treatment options and provide potential pharmacologic targets," notes Hinks.

The consortium is extending its genotyping efforts and plan to incorporate two new groups, from Norway and Canada, that have joined the Immunochip Consortium. "The increased sample size will enable us to stratify data to look for associations with different subtypes of JIA," says Hinks. "Understanding the underlying genetics will help refine the subtypes, and improve treatment choices and disease management."

David Killock

Original article Hinks, A. et al. Dense genotyping of immune-related disease regions identifies 14 new susceptibility loci for juvenile idiopathic arthritis. Nat. Genet. doi:10.1038/ng.2614 